\title{
Alexander G. Tartakovsky (2020): Sequential change detection and hypothesis-general non-i.i.d. stochastic models and asymptotically optimal rules
}

\section{CRC Press, 320 pp. \$140 (Hardcover), ISBN 978-1-498-75758-4}

\section{Claudia Kirch ${ }^{1}$}

Received: 8 February 2021 / Revised: 1 March 2021 / Accepted: 9 March 2021 /

Published online: 27 March 2021

(C) The Author(s) 2021

In many applications data is collected sequentially until a statistical decision can be made with sufficient confidence. Unlike in a posteriori analysis the sample length is not known a priori but depends on the previously observed data. Thus, the mathematical analysis of the random sample size until a decision is made often involves properties of stopping times. In classical sequential hypothesis testing a sequential approach allows to control both the type-I and type-II errors and methodology often aims at minimizing the expected number of observations necessary for this task. Another important topic in sequential analysis is the detection of structural breaks or change points in the data generating mechanism with applications in a variety of fields including quality control, finance, bio-medical signal processing or network analysis. In such surveillance problems the challenge lies in raising an alarm as soon as possible after a change occurs under some suitable constraints on the run length in the no change situation. Such change detection problems have a long tradition in statistics dating back to the 1930s. Whereas traditional approaches mainly consider simple, e.g. i.i.d. univariate data streams with known after-change distribution, the dawn of the data age requires methodology for more and more complicated data streams.

In view of these recent developments this book is the natural extension to the earlier book by Tartakovsky et al. (2014). It covers the case of possibly dependent discretetime models where compared to the previous book more complicated situations are covered such as composite post-change hypothesis with unknown distributional parameters or multi-stream scenarios. Especially the last setting has received a lot of attention throughout several communities, as more and more data is multivariate or even highdimensional. The book is mathematically rigorous and the reader is expected to have some previous knowledge of probability and statistics including 'a certain familiarity with complete and $r$-quick convergence, Markov random walks, renewal and nonlinear

Claudia Kirch

claudia.kirch@ovgu.de

1 Departmentof Mathematics, Otto von Guericke University, Magdeburg, Germany 
renewal theories, Markov renewal theory, and uniform ergodicity of Markov processes' (to quote the author from the preface of the book). Although some basic background in these fields is covered in the appendices, some previous experience is indeed useful. Therefore, the book is mainly aimed at researchers in this broader area of probability and mathematical statistics as well as graduate students wishing to specialize in sequential change detection. It is less accessible for practitioners wishing to get an overview over the area.

The main part of the book consists of eight chapters: The first chapter is set apart from the rest in that it is the only chapter not dealing with change detection but rather with classical sequential likelihood-based hypothesis testing controlling both the type-I error of a false alarm as well as the type-II error of a missed detection. While this topic is already discussed at length in Tartakovsky et al. (2014), the theory here is designed for multiple data streams, which is an important extension for modern applications.

The remaining seven chapters deal with various recent aspects of data detection methodologies: Chapter 2 gives a nice overview over different optimality criteria including several Bayesian and minimax criteria that are of interest for a variety of situations. The next chapter provides some discussion on asymptotic Bayes theory including extensions of Shiryaev and Shiryaev-Roberts detection statistics. As an example, results for Hidden Markov Models are obtained. A different viewpoint is taken in Chapter 4 , where the aim is to minimize the moments of the detection delay either pointwise or in a minimax sense, while controlling the probability of a false alarm within a window of given length. All of the methodologies from Chapters 1 through 4 accumulate the information from the data until a threshold is exceeded. Such approaches are optimal in terms of minimizing the expected number of necessary observations until detection. In Chapter 5 the focus is turned to maximizing the probability of detecting the change in a pre-specified time interval (after the change). Chapter 6 comes back to multiple data streams by extending the results from Chapter 3. In many applications, it is not only of interest to detect a change point but also to gain some insight into the type of change once it is detected. For example, in a multi-stream situation one might want to determine which one of the streams has actually changed. This is the problem discussed in Chapter 7, thus extending results from Chapter 10 of Tartakovsky et al. (2014). Finally, in Chapter 8 three potential applications are discussed in detail: Object track management in sonar systems, detection of traces of space objects as well as detection of unauthorized break-ins in computer networks.

In conclusion, I can recommend this book to researchers in mathematics and theoretical statistics who are interested in recent developments in sequential change detection.

Funding Open Access funding enabled and organized by Projekt DEAL.

Open Access This article is licensed under a Creative Commons Attribution 4.0 International License, which permits use, sharing, adaptation, distribution and reproduction in any medium or format, as long as you give appropriate credit to the original author(s) and the source, provide a link to the Creative Commons licence, and indicate if changes were made. The images or other third party material in this article are included in the article's Creative Commons licence, unless indicated otherwise in a credit line to the material. If material is not included in the article's Creative Commons licence and your intended use is 
not permitted by statutory regulation or exceeds the permitted use, you will need to obtain permission directly from the copyright holder. To view a copy of this licence, visit http://creativecommons.org/licen ses/by/4.0/.

\section{Reference}

Tartakovsky A, Nikiforov I, Basseville M (2014) Sequential analysis: hypothesis testing and changepoint detection. CRC Press, Boca Raton

Publisher's Note Springer Nature remains neutral with regard to jurisdictional claims in published maps and institutional affiliations. 